\title{
An Object-Based Approach to Implementing Distributed Concurrency Control
}

\author{
Steven S. Popovich \\ Gail E. Kaiser \\ Shyhtsun F. Wu \\ Columbia University \\ Department of Computer Science \\ New York, NY 10027
}

CUCS-051-90

20 November 1990

\begin{abstract}
We have added distributed concurrency control to the MELD object system by representing inprogress transactions as simulated objects. Transaction objects exploit MELD's normal message passing facilities to support the concurrency control mechanism. We have completed the implementation of an optimistic mechanism using transaction objects and have designed a twophase locking mechanism based on the same paradigm. We discuss the tradeoffs made and lessons learned, dealing both with transactions on objects and with transactions as objects.
\end{abstract}

Copyright (C) 1990 Steven S. Popovich, Gail E. Kaiser and Shyhtsun F. Wu

Popovich and $\mathrm{Wu}$ are supported in part by the Center for Telecommunications Research. Kaiser is supported by National Science Foundation grants CCR-9000930, CDA-8920080 and CCR-8858029, by grants from AT\&T, BNR, Citicorp, DEC, IBM, Siemens, SRA, Sun and Xerox, by the Center for Advanced Technology and by the Center for Telecommunications Research.

topics: Languages, Tools and Software Engineering; Distributed Databases 


\title{
An Object-Based Approach to Implementing Distributed Concurrency Control
}

\author{
Steven S. Popovich \\ Gail E. Kaiser \\ Shyhtsun F. Wu \\ Columbia University \\ Department of Computer Science \\ New York, NY 10027
}

20 November 1990

\begin{abstract}
We have added distributed concurrency control to the MELD object system by representing inprogress transactions as simulated objects. Transaction objects exploit MELD's normal message passing facilities to support the concurrency control mechanism. We have completed the implementation of an optimistic mechanism using transaction objects and have designed a twophase locking mechanism based on the same paradigm. We discuss the tradeoffs made and lessons learned, dealing both with transactions on objects and with transactions as objects.
\end{abstract}

\section{Introduction}

The MELD distributed programming language provides the application programmer with transaction blocks, sequences of statements that are serializable with respect to all other MELD code. MELD application code, whether or not within a transaction block, may include both synchronous procedure calls and asynchronous messages to the same or other objects in the same or different processes, possibly executing on different hosts. All code executed within a transaction block and any code executed by threads created directly or indirectly by the block are part of the same transaction.

The novel aspect of MELD's implementation of transactions is that the various entities enforcing the concurrency control mechanism are represented as simulated "objects" that communicate using MELD's normal message passing facilities. This notion of transaction objects permits significant reuse of design and code in the implementation of concurrency control mechanisms, reusing design/code from other parts of the object system implementation to support concurrency control and permitting substitution of one concurrency control mechanism for another, reusing much of the same design/code. We have implemented an optimistic concurrency control mechanism reusing the existing interprocess communication facilities, and have designed a twophase locking mechanism reusing the design for optimistic concurrency control.

The MELD distributed object system has been under development for several years, and a 
previous paper [8] presents the language and implementation as it was before transaction blocks were added. In a more recent paper [9], we describe the interactions between the three concurrency control policies now included in the language - serializable transactions for concurrency control across multiple objects residing in multiple processes, atomic blocks for critical sections on individual objects, and uncontrolled concurrency. This paper discusses our experience developing the first policy based on our notion of transaction objects.

We first give brief background information on the MELD object system, and then describe the optimistic concurrency control protocol we adapted to our transaction object paradigm. We explain the implementation of distributed optimistic concurrency control in terms of simulated objects and walk through a portion of a transaction processing demo program emphasizing the communication among these objects. We then discuss the experience gained and lessons learned through this effort. We go on to present our design for a distributed two-phase locking concurrency control protocol reusing the same interface, enabled by our transaction object paradigm. We briefly compare to related work, and conclude by sketching our ideas for future work.

\section{Background: The MELD Object System}

MELD is an experimental object system under development at Columbia University. The objectoriented programming language supports classes, strong typing of instance variables, active values, multiple inheritance, and separate compilation of modular units called features that bundle together related classes and objects. These facilities were developed for an early version of MELD [6, 7], without persistence, concurrency or distribution.

MELD now allows any class to be declared as persistent, and instances of persistent classes are stored in a B-tree database. Access to persistent and/or remote objects is through a network name service using a uniform syntax, so that application code need not "know', whether objects are stored locally or remotely, transiently or persistently. The MELD programming language is now concurrent, with new threads of control created by asynchronous messages and also effectively by synchronous procedure calls, which are implemented internally by an asynchronous message pass followed immediately in the sending thread by a statement waiting for the return message. Multiple threads may execute concurrently in the same object as well as in different objects.

MELD is implemented by translating the MELD code into C. The compilation system consists of a preprocessor mpp (which resolves inheritance), a compiler mc (which generates $\mathrm{C}$ code and feature symbol tables), and a linker $\mathrm{ml}$ (for combining separately compiled features). The runtime system consists of a kernel (supporting scheduling, persistence, transaction objects, etc.) linked into all MELD applications, and a separate nameserver program that must be running on every host where a MELD application executes. Arbitrary $\mathrm{C}$ code may be called from within MELD code, and is also linked by ml. The compilation system contains approximately 9600 lines of C, 3800 lines of Yacc, and 1100 lines of Lex. The runtime kernel includes approximately 11,300 lines of C, 700 lines of Yacc, and 200 lines of Lex (some of this is for the MELD Debugger [5], not discussed here). The name server consists of approximately 2400 lines of C. 


\section{Background: Concurrency Control Algorithms}

Our first application of the transaction object concept implements the multi-version parallel validation (MVPV) optimistic concurrency control algorithm. MVPV, presented in a paper by Agrawal et al. [1], is a variant of the original optimistic concurrency control algorithm devised by Kung and Robinson [10]. Only short descriptions of these algorithms are included here; the full details are in the papers. ${ }^{1}$ Our second application of transaction objects, only a design at this point, is to the conventional two-phase locking concurrency control algorithm (2PL) [2]. We assume that the reader is familiar with 2PL. Both MVPV and 2PL employ standard distributed commit protocols, involving a coordinator in the process where the transaction is initiated and cohorts in every process where the transaction executes; we also assume the reader is familiar with such commit protocols.

Optimistic concurrency control mechanisms are so named because they do not use locking to protect objects from concurrent access. Instead, they make the "optimistic" assumption that there will not be any conflicts among transactions, and then check when each transaction completes whether this assumption was in fact true. When a conflict is detected with an earlier transaction, the later transaction is rolled back and restarted; otherwise, it is committed.

Each optimistic transaction is implemented in three phases, a read phase where objects are read and shadow copies of objects are written, a validation phase where the runtime system checks whether or not the "optimistic" assumption was valid, and a write phase for validated transactions where the shadow updates are copied to the actual objects. During the read phase, a transaction accesses any resources it needs without waiting for locks, but instead records the sets of objects read and written (the transaction's read set and write set). The transaction is validated when it finishes by comparing its read set and write set against other previously completed transactions. The read set must not conflict with the write set of any other transaction that validated since this transaction began, and the write set must not conflict with any other transaction that has entered validation but not yet completed its write phase.

A simple pseudo-code description of Kung and Robinson's original optimistic algorithm is given in Figure 3-1. This version assumes that only one transaction is validating at any given time; their paper also presents an algorithm for validating transactions in parallel. The tbegin section is executed at the beginning of each transaction to initialize the transaction's internal data. Then the transaction executes, and collects its read and write sets. The tend section runs when the transaction is ready to commit, and performs validation.

Kung and Robinson use parentheses "(...)" as begin-end blocks and angle brackets "<...>" as critical section delimiters, indicating the portion of the pseudo-code that must be executed serially with respect to other transactions. We have omitted portions of their algorithm having no impact on concurrency control.

The MVPV validation algorithm is based on two transaction number counters: a commit transaction number counter $(c t n c)$ and a visible transaction number counter $(v t n c)$. ctnc counts

\footnotetext{
${ }^{1}$ According to one of the authors of the MVPV paper, Soumitra Sengupta, ours is the only implementation of that algorithm.
} 


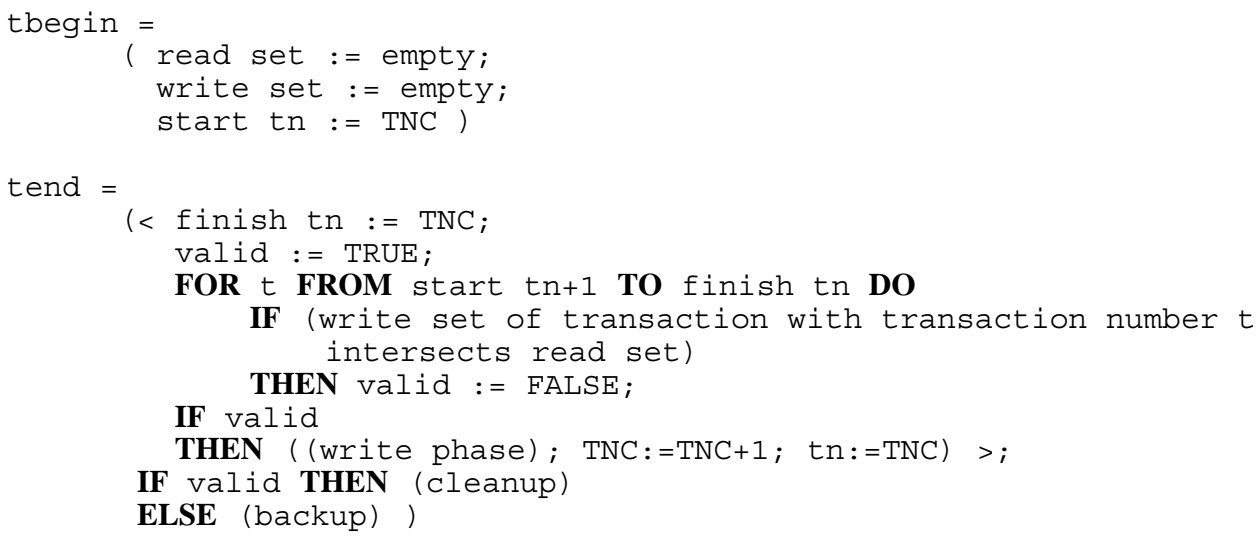

Figure 3-1: Basic Optimistic Concurrency Control Algorithm

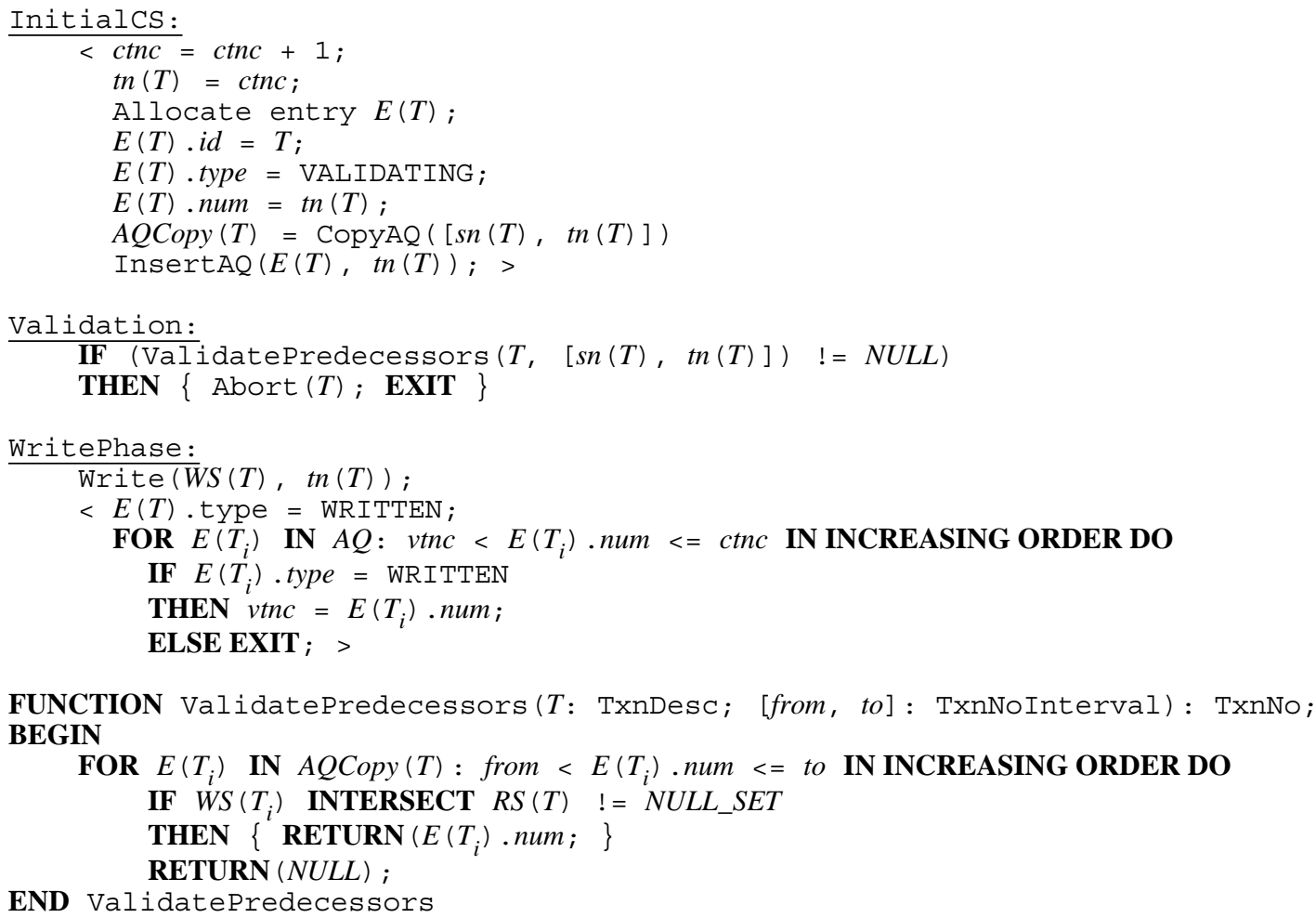

Figure 3-2: Multi-Version Parallel Validation Algorithm

the transactions that have entered validation, while vtnc holds the highest transaction number whose results are visible to other transactions (i.e., its write phase has completed). This is the latest transaction that has completed its write phase and has no transaction before it that has not also finished. MVPV combines optimistic concurrency control with multiple versions [12], so read operations of a transaction access the last visible version written prior to the start of the transaction. Validation is thus unnecessary for read-only transactions, which obtain a consistent but not necessarily up-to-date view. For applications with many read-only transactions, MVPV has an advantage over pessimistic (locking) schemes as well as the original optimistic scheme, because of the very low overhead for read-only transactions. A pseudo-code description of the MVPV algorithm is given in Figure 3-2. 
A transaction $T$ begins (not shown) by being assigned a start number $(\operatorname{sn}(T))$ that defines the highest transaction number whose results are visible to $T$ (the current $v \operatorname{tnc}$ ). As $T$ continues its read phase, its read $(R S(T))$ and write $(W S(T))$ sets accumulate. Reads of data not yet written by $T$ see the last version of the object written before $\operatorname{sn}(T)$, while writes create a new shadow version specific to $T$ as in the original optimistic algorithm, which is then seen by subsequent reads by $T$. Many of these versions may exist at any time, since there can be one for each active transaction. There may also be more than one visible version of the same object; old versions must be kept until such time as there are no transactions that may reference them. Eventually $T$ ends (from the application program's point of view), entering its validation phase.

The initial critical section, InitialCS, then assigns the transaction its transaction number $\operatorname{tn}(T)$ (the incremented $c t n c$ ), which determines the serialization order of transactions, and sets up the transaction's entry in $A Q$, the active queue. AQ contains an entry for every transaction that has entered validation prior to this one, for the transaction to validate against. Actually, each transaction keeps a partial copy, AQcopy, of $A Q$ to avoid problems with concurrency on this important data structure as they validate in parallel. Entries in $A Q$ older than the earliest $s n$ of any running transaction will never be considered in validation, and so may be discarded. This keeps the space taken by $A Q$ from growing without bound as long as all transactions eventually terminate.

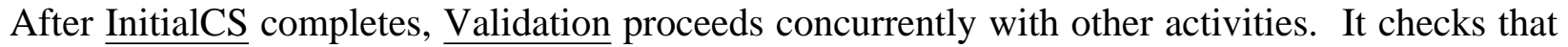
there is no transaction with a transaction number $t n$ after $\operatorname{sn}(T)$ that has written a new version of any data read by $T$. (Concurrently validating transactions never cause ambiguities in this ordering, since $t n$ 's are assigned serially by InitialCS.) If such a transaction exists, $T$ must abort, since its results are based on old data and may not be consistent with the new data. This is unnecessary when the current transaction is read-only, because such a transaction may always be considered to have finished at its start time without affecting the serialization of other transactions. If $T$ validates successfully and can commit, it enters its WritePhase, and a final critical section marks $T$ as having written its results and updates vtnc. $T$ 's new versions now become public, and are visible to new transactions if the new $v t n c$ is at least $\operatorname{tn}(T)$. If not, they will become visible at a later time, when this condition becomes true.

We have presented the MVPV algorithm in centralized rather than distributed form; the distributed form is described in detail in Agrawal et al.'s paper. The transformation to the distributed form involves consideration of the range of start numbers and transaction numbers for different cohorts, or sites participating in the transaction. The range is determined by a coordinator by combining information from all the cohorts, and the validation algorithm is executed at each of the cohort sites of a transaction with conflicts detected over the full range rather than using any of the various local start and transaction numbers.

This process requires a three-phase commit protocol instead of the usual two-phase protocol; an additional phase is added at the beginning to calculate the range. The three phases are as follows:

1. Determine global transaction numbers - The coordinator detects termination of a transaction and asks each cohorts to send back its local numbers for the transaction. Each cohort replies with its local start number and local transaction number. The 
coordinator determines the global start number and global transaction number by taking the minimum of the local start numbers and the maximum of the local transaction numbers (respectively).

2. Validate - The coordinator sends the global start and transaction numbers to all cohorts. Each cohort replies, informing the coordinator whether or not the transaction could be validated at the cohort with the global numbers.

3. Commit or Abort - If the transaction was validated at all cohorts, the coordinator tells the cohorts to commit, and they update their current versions of all objects involved in the transaction from their shadow versions of those objects. Otherwise, the coordinator tells the cohorts to abort, and they discard their shadow versions for the transaction.

This description has been somewhat simplified from Agrawal et al. to avoid discussing technical details such as the safety factor that is applied to the global transaction number. For a complete description, see their paper.

\section{Implementation of Distributed Optimistic Concurrency Control}

MELD's transaction subsystem implements the distributed form of the MVPV algorithm. The main technical decision that we made in adapting the algorithm was the granularity at which to apply it: to entire objects or to individual instance variables within objects. We opted for multiversion variables, rather than objects, because of the increased concurrency this allows. Ramifications of this decision are discussed in Section 6.

We represent transactions as $\mathrm{C}$ structures, as first-class MELD objects are also represented internally, but the $\mathrm{C}$ structures do not possess all the attributes of MELD object structures, and cannot be treated as MELD objects by the runtime system; thus we refer to transaction objects as "simulated", even though the same message passing facilities are employed. C declarations for the major data structures of the transaction implementation are shown in Figure 4-1.

A transaction structure may represent either a coordinator or a cohort object. Some fields are used only in coordinators, some only in cohorts, but most are used in both kinds of objects. Had we implemented transactions as first-class MELD objects, we would most likely have defined two subclasses of a "Transaction" class, namely "Coordinator" and "Cohort", rather than use only one data structure for both. Since transactions are not first-class MELD objects, but only C structure simulations, and we needed to be able to treat both coordinator and cohort objects as transactions without distinction in some cases, we employed a single structure.

A transaction object consists of:

- A type that distinguishes between "real" and "fake" transactions. MELD code initiated directly or indirectly by a transaction is part of the "real" transaction. All other MELD code, running outside any transaction, uses the notion of "fake" transactions to keep track of what it has read and written so that "real" transactions can validate against these operations. "Fake" transactions cannot abort, but they may cause "real" transactions to abort due to conflicts. The need for "fake" transactions is explained in Section 6.

- A global host (g_host) string that contains the name of the machine where the 


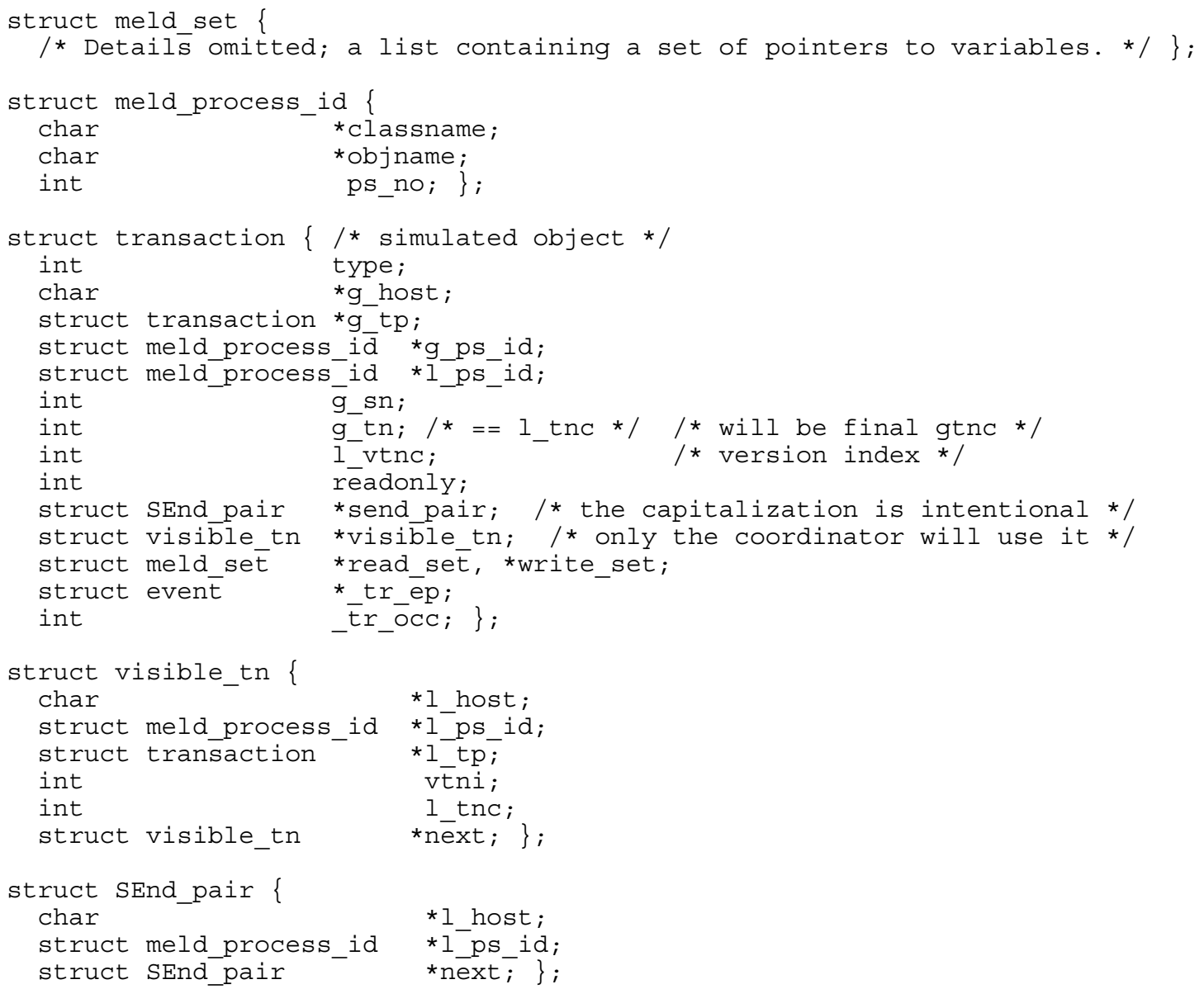

\section{Figure 4-1: Transaction System Data Structures}

coordinator of this transaction lives. This is the same in both the coordinator and the cohorts of a transaction. It exists only for historical reasons; it has been superseded by the g_ps_id field (see below).

- A global transaction pointer (g_tp) that points to the coordinator object in both the coordinator (where it points to itself) and the cohorts. This is not a valid pointer except in the process where the coordinator lives, but it serves as identification and as a means of distinguishing coordinator objects from cohort objects.

- A global process id (g_ps_id) and a local process id (1_ps_id) that record the object names that the coordinator (global) and cohort (local) objects, respectively, are registered under with the name server. Before an object can receive messages from remote objects, it must be registered; when messages are sent to the coordinator or a cohort, they are sent to these names. This structure and its use are described in more detail below.

- A global start number (g_sn), a global transaction number (g_tn), and a local visible transaction counter (1_vtnc) that serve as the variables of similar names in the distributed MVPV algorithm. g_sn is the minimum of all cohorts' local start numbers, and g_tn is the maximum of the local transaction numbers, disregarding safety factors. These two fields are used only by coordinators. I_vtnc is used by cohorts; it is the local start number for the transaction. Public versions created before l_vtnc are visible to the cohort. 
- A readonly field that is true if the transaction has not written any data. In the cohorts, this field is not used; a cohort is read-only if its write set is empty. In the coordinator, this field summarizes the information in all the cohorts. When a readonly transaction terminates, no validation need be done.

- The send-end pair list (send_pair) that exists only in the coordinator and keeps track of all of the threads running within the transaction. Its job is termination detection; a simple algorithm is used for this. The name comes from the messages used in the algorithm, send_begin_msg and send_end_msg. Every time a MELD method is called within a transaction, creating a new thread, a send_begin_msg is sent to the coordinator. The coordinator then adds a SEnd_pair element to this list to represent the thread. Every time a method terminates within a transaction, a send_end_msg is sent, and the corresponding element is removed from its coordinator's list. $^{2} \mathrm{~A}$ transaction finishes when its send_pair list becomes empty, signifying that all methods (and thus all threads) within the transaction have terminated. The distributed commit protocol then begins.

- A visible transaction number structure list (visible_tn) that keeps an entry for each process that has a cohort in the transaction. Only coordinators have this field. It is used during the commit protocol.

- A read_set list and a write_set list that keep track of the instance variables that have been read and written, respectively, by the transaction within the local process. These are found only in cohorts.

- Other information (_tr_ep and _tr_occ) that is needed in case of an abort in order to restart the transaction, but the format is not relevant to this discussion. These are only in the coordinator, as individual cohorts cannot restart independently of the coordinator.

A meld_process_id is an address for a process in which a transaction object resides, as understood by the MELD name service. It consists of a classname and an objname unique within the class. The meld_process_id is used whenever it is necessary to send a interprocess message from one transaction object to another. It contains the classname and objname strings of an "object'" representing the coordinator's process (in the g_ps_id field) or the local process (in the I_ps_id field). We use process identifiers, rather than individual transaction object identifiers, to avoid the overhead associated with notifying the name service of the creation and destruction of each transaction. In use, a meld_process_id is always paired with a transaction pointer (valid in its own process only). The name service delivers each message to its destination process, and within that process, the transaction pointer provides a local dispatch address. The classname is fixed for all transactions ${ }^{3}$, and the objname is a printable form of the process identifier on the local machine. The ps_no field holds the process identifier as a number. It is present for historical reasons and is no longer used, except in debugging.

\footnotetext{
${ }^{2}$ Actually, any element with the same 1_ps_id, it does not matter unless a send_end_msg arrives before a matching send_begin_msg. The termination algorithm, designed for local area networks where that case does not occur, does not handle it well.
}

${ }^{3}$ Since a meld_process_id identifies a process to the name server, the word "SERVER" is used as the classname. 
In MELD, all message passing outside of the local process goes through the name service, which looks up the name and forwards the message to the proper host by means of a server-to-server protocol, where it is delivered to the destination process by the remote server. This is true for both ordinary messages between first-class MELD objects and for the special messages between transactions; the only difference is in how they are treated in the network message handler once they arrive at the local MELD process. Non-transaction messages, including both ordinary and return messages, are delivered directly to the named object. (Return messages are the replies, usually containing a return value, sent back to the caller at the completion of a synchronous RPC.) Transaction messages are sent to the particular transaction "object' whose pointer (in the named process) is included in the message.

The visible_tn structure holds information that enables the coordinator to find each of its cohorts when it runs the commit protocol. The coordinator has a list, each element of which includes the local hostname of the cohort and its message passing address (its (1_ps_id plus its local transaction pointer). During the commit protocol, validate_request messages must be sent to all cohorts, validate_return messages received from them, and either real_write or destroy_version messages sent back, depending on whether the transaction commits or aborts.

In addition to the necessary addresses, the visible_tn structure also contains information about the transaction number variables maintained by the MVPV algorithm at each site. These are used to derive the global start number and global transaction number of the transaction: a multiprocess transaction is considered to have started at the earliest of its local start numbers (vtni), and to have entered validation at the latest of its local transaction numbers (1_tnc), plus a small safety factor. (g_sn holds this earliest start number, and g_tn holds the latest transaction number (including the safety factor), in the transaction structure.) This leads to unnecessary aborts in situations where the current and visible transaction numbers are not in reasonably close synchronization among cohorts. We have worked out a solution where, whenever a transaction finishes, these variables are synchronized in all of the participating processes in the transaction. We describe the problem and its solution in detail in Section 6.

Figure 4-2 gives the headers and brief descriptions of each of the operations ("methods") on transactions. All operations are implemented as $\mathrm{C}$ functions, with local optimistic concurrency control-related operations on cohort objects called by compiler-generated $\mathrm{C}$ code within the local process, and global distributed commit-related operations on coordinator and cohort objects invoked by sending the appropriate messages using MELD's normal interprocess message passing facilities.

\section{Example of MELD Transaction Management}

We now show how transaction objects are used in MELD by walking through an example transaction. Our example comes from a toy stock trading application, with several distinct types of MELD objects: stocks, stock portfolios, checking accounts, and users. To buy a stock, a user sends a buy message to the user's stock portfolio. This method, implemented as a transaction block, sends a quote message to the stock to get its current price and a withdraw message to the user's checking account to get the money to buy the stock. It then updates the user's portfolio to reflect the stock that was purchased. 


\section{Local Optimistic Concurrency Control Operations}

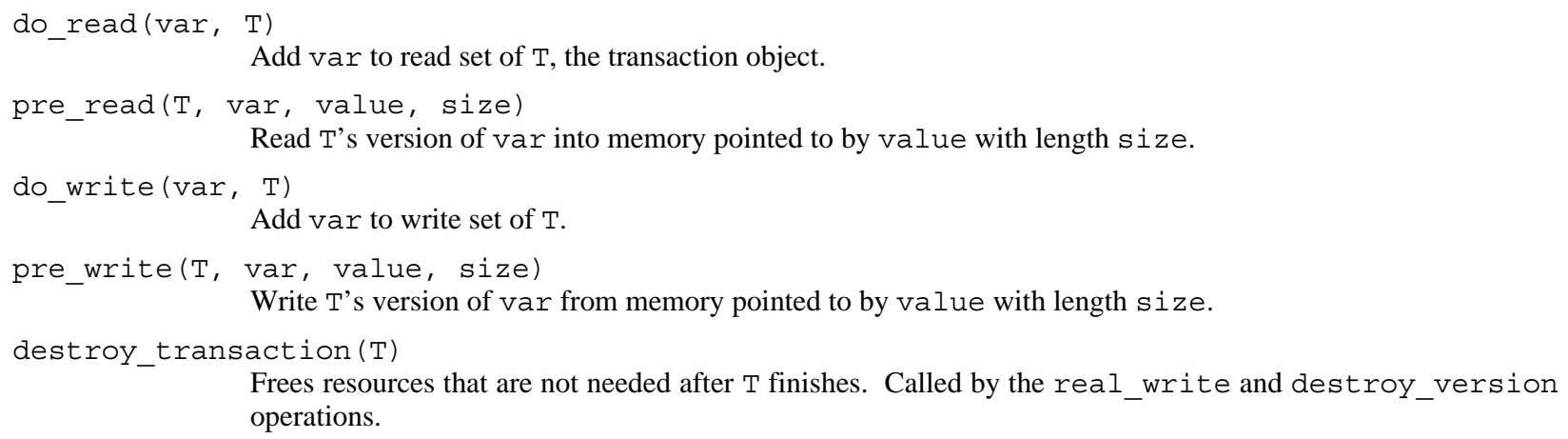

These misleading names exist for historical reasons. For example, do_write prepares for pre_write and is called before it.

\section{Global Distributed Commit Protocol Operations}

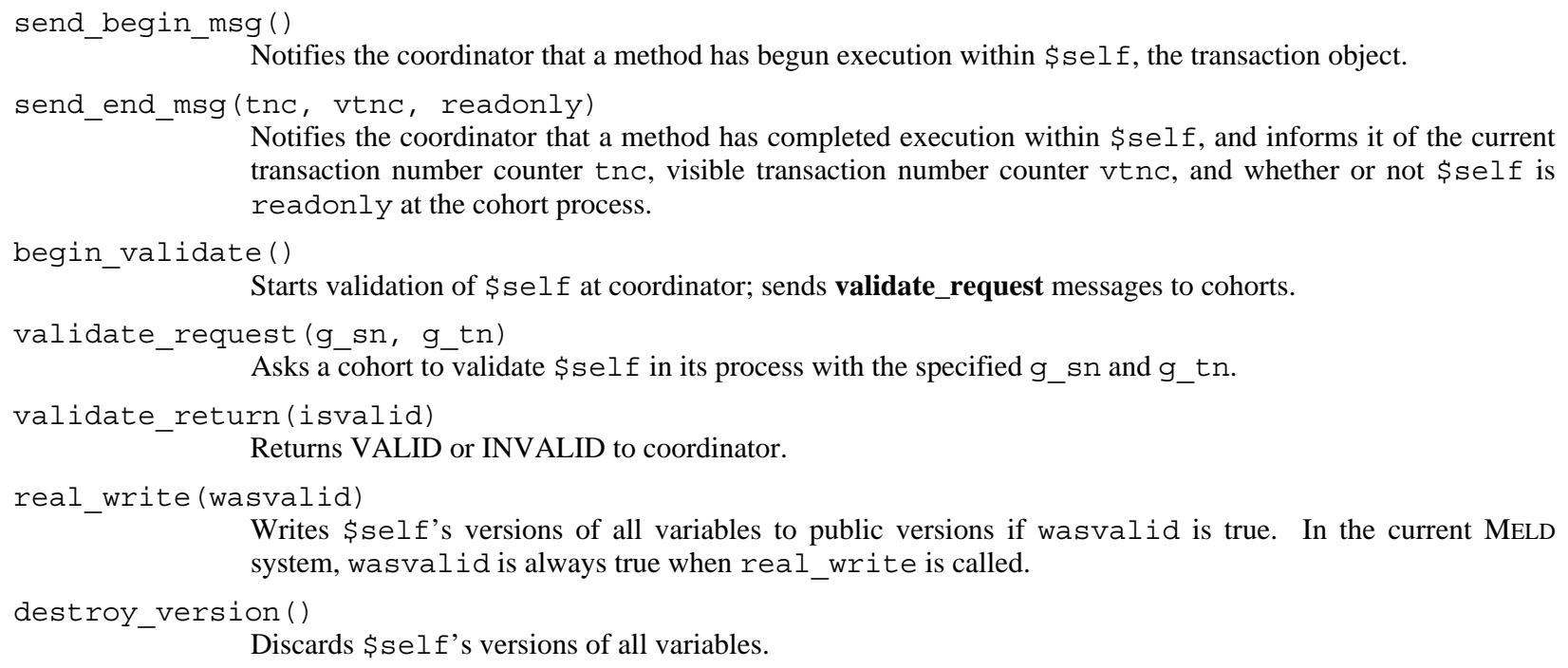

Figure 4-2: Operations on Transaction Objects

This activity thus involves three objects, which must be kept consistent, and up to three cohorts since the objects may reside in different processes (which may execute on different hosts), as depicted in Figure 5-2. Figure 5-1 shows the relevant portion of the MELD code for these methods. Due to space limitations, the code for demo-related messages has been removed, leaving only the basic checking account and stock manipulation code.

We assume that the user already owns some shares of IBM and wants to buy 10 more. To do this, the user types a message on the terminal that causes the user object to send the message buy $(10, " I B M ")]$ to the corresponding stock portfolio. The events from this point onward are depicted in Figure 5-2; the numbers below correspond to those on the arrows in the diagram.

1. The buy message arrives at the portfolio object and the corresponding method is invoked. Entering the transaction block (marked by "<<...>>" in the code) has the side effects of creating a coordinator object and a cohort object in the current process, and then the cohort sends the send_begin_msg to the coordinator. (In this example, names and code written in MELD appear in typewriter font, and 


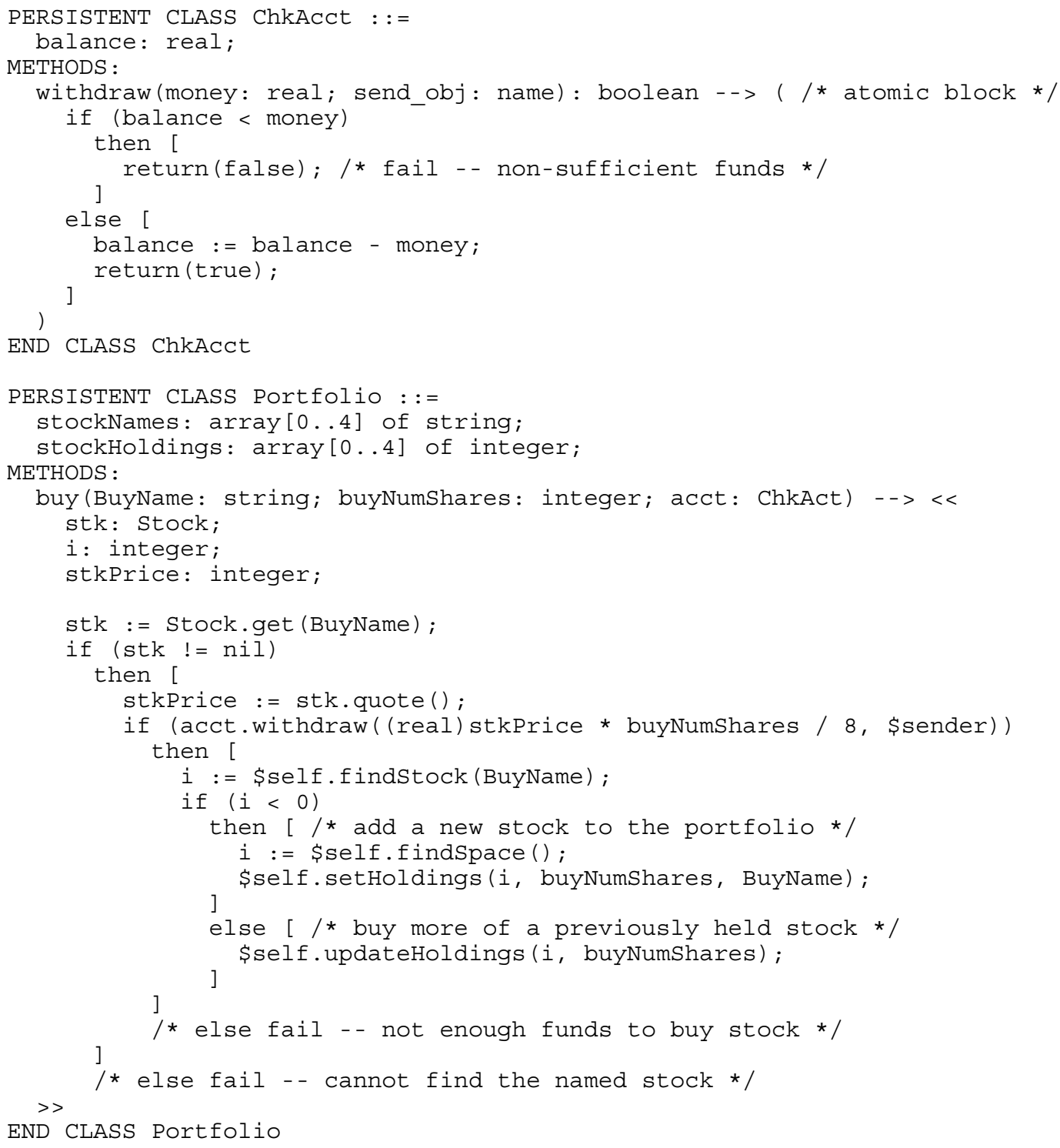

Figure 5-1: MELD Transaction Example: Code

names and code implementing MELD appear in boldface. In figures, all code appears in typewriter font.) When the send_begin_msg is received by the coordinator, an element is added to its send_end_pair and visible_tn lists to represent this thread of control.

2. The portfolio then gets a handle on the persistent object representing the stock named "IBM", which involves an exchange of messages with the name server (not shown in the diagram), and sends a quote message to the stock. This message implicitly carries the transaction identifier, which is the address of the coordinator object (meld_process_id). When the quote method is invoked, this has the side effects of creating a local cohort for the transaction in the stock object's process and sending a send_begin_msg message back to the coordinator. Again, when this is received by the coordinator, an element for this thread is added to the send_end_pair and visible_tn lists. (Actually, there is only one element in the visible_tn list per cohort rather than per thread, but there is no distinction in this 


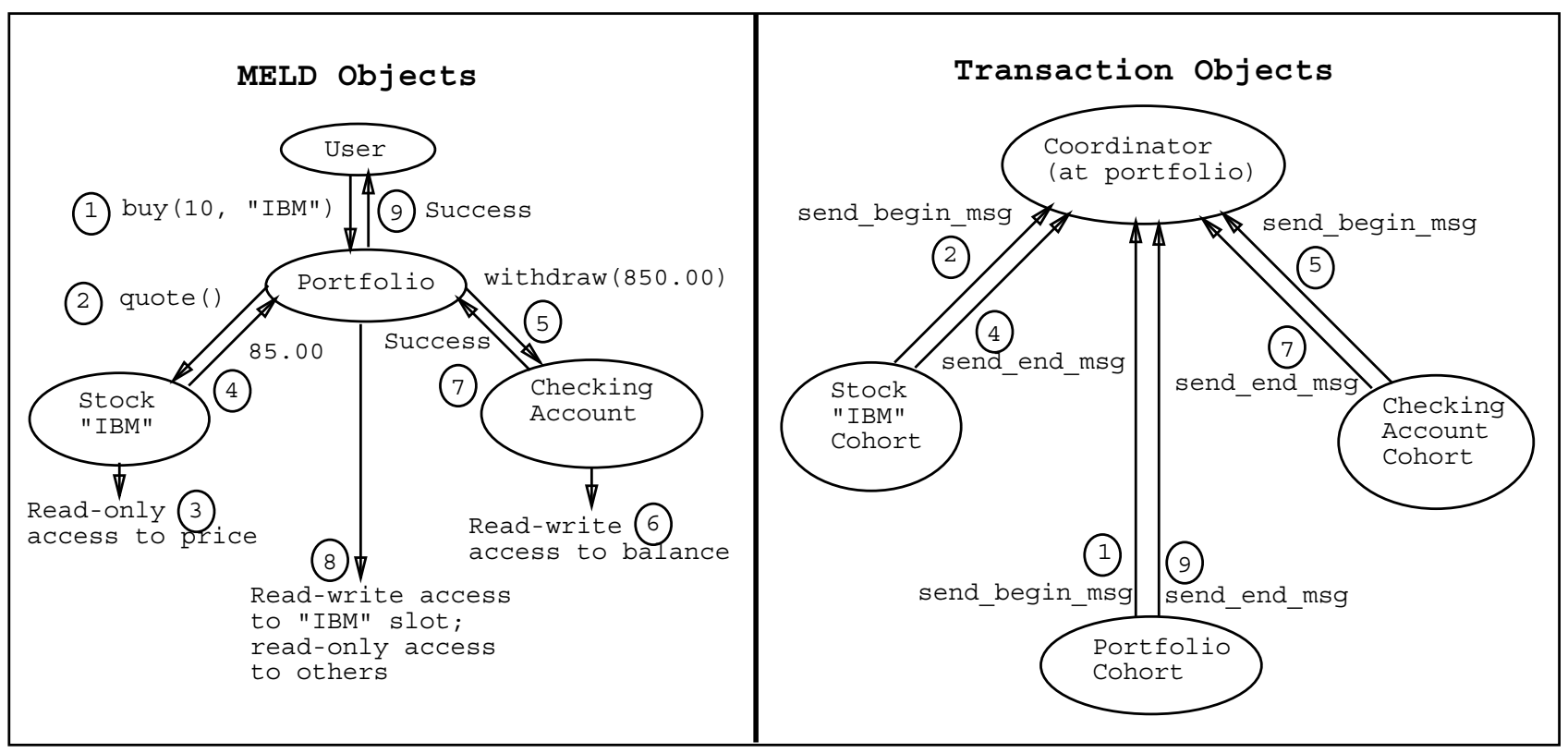

Figure 5-2: MELD Transaction Example: buy (10, "IBM" )

example.)

3. When the quote method (not shown) reads the price instance variable of the stock object, the do_read operation is invoked to add this variable to the cohort's read_set and then the pre_read message accesses the appropriate version of this instance variable (the version written by the last visible transaction before the start time of the cohort, its vtnc).

4. The quote method sends a message returning the stock's price per share (for example, \$85.00) back to its caller, the stock portfolio. As a side effect, a send_end_msg message to the coordinator is also generated; this lets the coordinator know that the thread in the stock object has completed, which is recorded by removing the corresponding send_pair element from the send_end_pair list (but the element corresponding to the stock object's cohort remains in the visible_tn list). When the return message arrives, the buy method in the portfolio object continues.

5. The next step is to withdraw the money to buy the stock, so buy sends a withdraw message to the checking account object. When the withdraw method is invoked, it has the side effects of creating a cohort object in the checking account's process, since the transaction has not previously involved that process, and sends a send_begin_msg to the coordinator object. The withdraw method checks that the balance is sufficient; in doing this, the do_read and pre_read operations add the balance instance variable to the cohort's read_set and read the appropriate version of the balance value for the transaction, respectively.

6. withdraw then subtracts the amount specified from the balance and writes the new balance back; the side effects are do_read, do_write and pre_write operations for balance. The do_read does nothing, since balance is already in the read_set, do_write adds balance to the write_set, and pre_write creates the appropriate new shadow version for this instance variable. 
7. The withdraw method concludes by sending a message back to the portfolio indicating that the withdrawal succeeded, and the buy method continues again. This return message has the usual side effect of a send_end_msg message to the coordinator, to tell it to remove this thread from its send_end_pair list.

8. The buy method next finds the slot for "IBM" in its portfolio and increments the number of shares by 10 . This involves the four optimistic concurrency control transaction operations for adding the number of shares to the read set, actually reading the number of shares, adding this variable to the write set and writing its shadow version. Since the "IBM" slot is found via direct linear search, the variables (array elements) holding the names of all of the stocks before "IBM" in the portfolio were also read, with corresponding do_read and pre_read operations invoked as side effects.

9. Finally, when buy is finished, it sends a return message to the user object to say that it succeeded; this results in a send_end_msg to the coordinator. After processing this message, the coordinator realizes it has no more active threads, and so begins the commit protocol.

MELD's two-phase commit protocol resembles the last two phases of Agrawal's three-phase protocol, and is implemented by coordinator and cohort objects as follows:

1. The coordinator determines the global start number $\mathbf{g} \_\mathbf{s n}$ and global transaction number g_tn by taking the minimum of the local start numbers and the maximum of the local transaction numbers, plus a small constant safety factor. These numbers have already been collected, as the vtni and l_tnc, respectively, of each cohort represented in the visible_tn list. vtni was set by the earliest send_begin_msg message from the cohort, and l_tnc by the latest send_end_msg from the same cohort process (these values are piggybacked on all the termination detection messages from cohorts).

2. The coordinator sends this pair of numbers to all cohort objects in validate_request messages.

3. The cohorts reply with validate_return messages, which tell the coordinator whether or not the transaction could be validated at the cohort with these global transaction numbers.

4. If the transaction was valid at all cohorts, the coordinator then sends the cohorts a real_write message, and they update their current versions of all objects involved in the transaction from their shadow versions of those objects.

5. Otherwise, the coordinator sends the cohorts a destroy_version message, and they discard their shadow versions for the transaction.

To support recoverability, acknowledgments would be required from every cohort to complete the second phase, but this is not addressed in this paper. The commit protocol messages for our example are shown in Figure 5-3. 


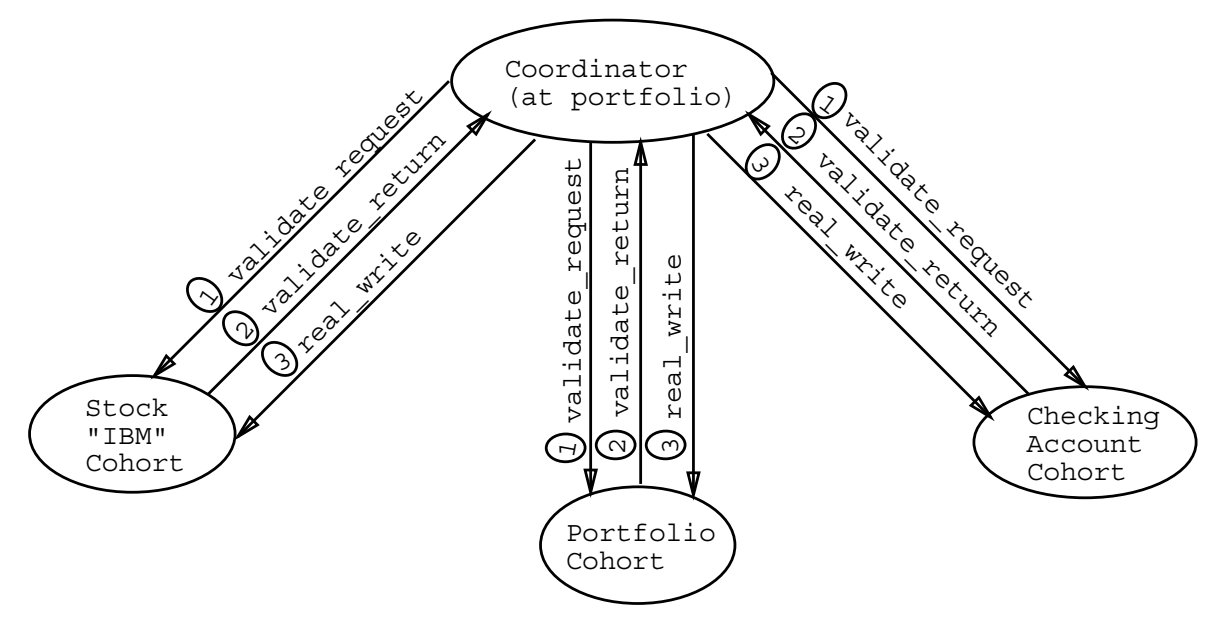

Figure 5-3: MELD Transaction Example Commit Protocol

\section{Experience with Transactions on Objects}

In Section 4, we touched on a problem caused by running an optimistic concurrency control algorithm across processes with widely differing usage patterns. Here, we describe that problem in detail and discuss several other important properties of transactions on objects and the difficulties they caused in the MELD implementation.

Consider the case where the participants in a transaction are a server and a client using the service, e.g., a "bank" process providing access to checking account objects and a "user" process accessing a particular checking account. In this case, since the client only occasionally initiates a transaction with the server but the server may continually be responding to many clients' requests, it is easy for the server's transaction numbers to become much larger than the client's.

For example, a client and a server may have completed a transaction $T_{1}$, after which the client became inactive for a long period. This situation is shown in Figure 6-1. When the client initiates another transaction, $T_{2}$, with the server at time $T_{2 S}$, the client's transaction number counter is be only 18 while the server's is 4096 . If $T_{2}$ begins validation at $T_{2 V}$ after a small increase in the transaction numbers at both sites (bringing the client's transaction number to 19 and the busier server's transaction number to 4100), the validation algorithm may find a conflict between $T_{2}$ and some earlier $T_{1}$, although no conflict actually exists. This is due to taking the global start number as the earliest (18) of the local start numbers and the global transaction number as the latest (4100) of the local transaction numbers, plus a safety factor (omitted here and in Figure 6-1), and validating against this wide range of transaction numbers at all cohort sites. The validation algorithm sees the older transaction $T_{1}$ on the same account at the server as being in conflict with the more recent $T_{2}$, although they actually executed in serial order. This perceived conflict appears because the server's $T_{l V}, 1029$, is within the distributed validation range [18-4100].

Our solution to the problem of gross desynchronization of the transaction number counters exploits the transaction number information carried by validate_request messages from the coordinator to cohorts during the commit protocol. Instead of simply validating against the $\mathbf{g} \_\mathbf{s n}$ 


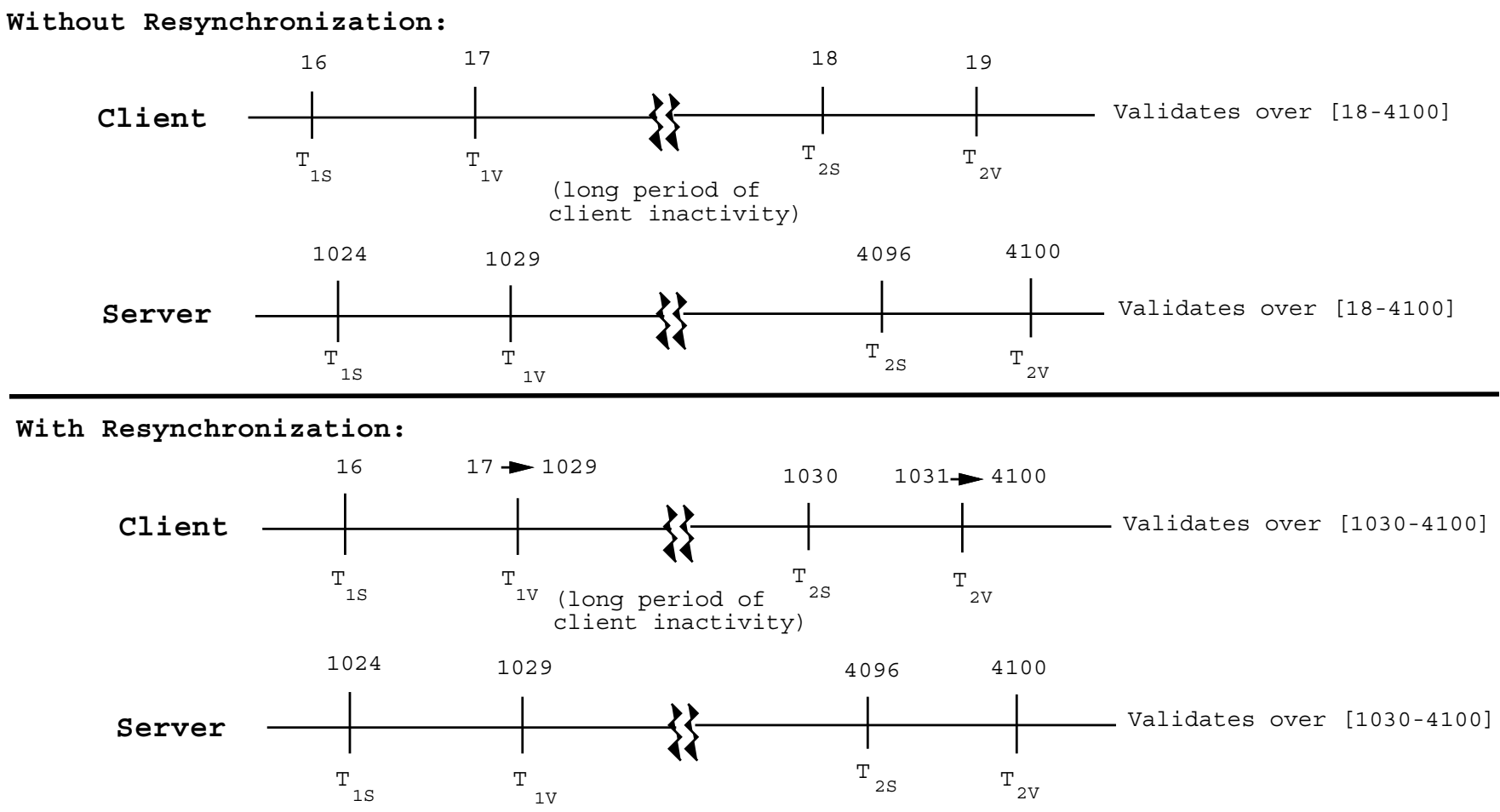

Figure 6-1: Example of Transaction Number Desynchronization

and $\mathbf{g}$ _tn provided, each cohort also updates its transaction number counter ctnc to the maximum of $\mathbf{g}$-tn and the current value. As the second part of Figure 6-1 shows, this results in $T_{2}$ validating against the range [1030-4100] while the server's $T_{1 V}$ remains 1029 , so the server no longer (erroneously) detects a conflict between $T_{1}$ and $T_{2}$.

This approach does not entirely prevent the problem of desynchronization, since a long-inactive client would remain out of synchronization until such time as it attempts to validate a transaction, and in fact, such a transaction is likely to abort due to being out of synchronization. But earlier transactions from the same client are guaranteed not to cause validation conflicts, since the client's and the server's transaction numbers were in agreement after the previous transaction, and in any case, even if the first transaction from an inactive client does abort unnecessarily, it will also synchronize the client with the server so that when the transaction restarts, the new incarnation will not encounter the same problem.

Subsequent transactions will remain reasonably well synchronized until the next long inactive interval, since the participants resynchronize at the end of each transaction. To prevent the problem entirely, it would be necessary to update all processes' local transaction number counters (ctnc's) at the end of every transaction; this would not only be prohibitively expensive in a large system with many processes, but is impossible to guarantee in a distributed environment where new processes may be created on any host at any time. Note that the reason for synchronizing transaction numbers at the end rather than the beginning of each transaction is that the complete set of participants in a particular transaction is not known until termination is detected and validation begins.

In addition to the desynchronization problem, we discovered several other problems that 
occurred in the MELD implementation due to simply having transactions running on objects, rather than to treating the transactions as objects themselves. Assumptions that had implicitly been made during the initial implementation of MELD as a non-transactional object system were broken by the addition of transactions. For example, in our original implementation, all messages were treated identically. There was no difference between synchronous procedure calls and asynchronous messages; the compiler simply generated additional code to wait for the return message in the first case, but not the second. There was also no difference between these return messages and any others. It seemed like a nice, clean design.

When we added transactions to the MELD system, the message passing code was modified to transmit the current transaction identifier (the meld_process_id of the coordinator's process, plus a pointer to the coordinator "object'" in that process) along with the message, and to create a cohort for the transaction in the remote process if one did not exist already, extending the transaction to that process. This caused a very hard-to-find bug, where if a method written as a transaction were called synchronously, later changes to data in the calling method would fail for no obvious reason. The bug turned out to be due to our uniform treatment of messages; the return message from the synchronously-called transaction was propagating the transaction identifier back to its caller and creating a cohort for it there, after the transaction had already "terminated" and started its commit protocol. Once this bug was found, fixing it was easy, though at the cost of treating return messages specially.

Another problem with transactions on objects deals with the treatment of interactions between transactional and non-transactional code on the data in the objects. In systems like Avalon [4], entire objects are declared as "atomic", meaning that every call to one of the object's methods is a separate transaction. In systems that support transactions across multiple objects, this problem must be dealt with somehow. Since concurrency control algorithms do not generally consider the possibility of non-transactional access to shared objects, that is, access by code that does not follow the conventions of the concurrency control mechanism. Non-transactional access is desirable in cases where performance is more important than strict data consistency, e.g., usage statistics kept by a network management application [11].

In a system containing both transactions and non-transactional code, however, the nontransactional code must respect some of the transactions' concurrency control conventions. If the transactions use a locking algorithm, all code must acquire a lock before accessing an object for writing, or the transactions' integrity may be compromised. For reading, on the other hand, no lock need be obtained by non-transactional code. Without a read lock, the non-transactional code may see an inconsistent version of the data, but it cannot violate the consistency of the transactions as it could by writing without a lock.

In an optimistic algorithm, any code that fails to keep at least a write set for validation purposes may cause the same problem. Our solution was to create "fake" transactions for nontransactional code. The transaction objects for "fake"' transactions look like any others except for their type field, and accumulate read and write sets in the same way as the "real" transactions do. They are not, however, validated when they terminate; their results are written to the actual objects (not shadow copies) without regard to any conflicts that may have occurred. Thus, non-transactional code may still corrupt other non-transactional code arbitrarily; we make 
no guarantees about that. What "fake" transactions do is allow "real" transactions to validate against them and abort (and restart) in case of conflict. This protects the "real" transactions against having their data corrupted by concurrent non-transactional code, while "fake" transactions still have a performance advantage, since they require no validation. This interoperability among MELD's three concurrency control policies (no control, atomic blocks and transactions) is explored in detail elsewhere [9].

A final problem discovered during our experience implementing transactions on objects is in dealing with the creation of objects by transactions. Object creation is currently independent of transactions; that is, when an object is created within a transaction, it is created immediately, and may appear in the name space before the transaction commits. In the case that a transaction creates an object and aborts, the object is not deleted, but remains instead as a "content-free" object, with all of its variables set to null values.

This problem is a side effect of our decision to treat instance variables as the granularity of detecting conflicts among concurrent transactions, to allow greater concurrency than would be possible if entire objects were considered as units by the concurrency control algorithm. Since MELD has no versions of objects, but only of instance variables within each object, there was no place to put a notation that the object should appear in the name space only during the transaction's write phase, or that it should be deleted if the transaction were to abort. This problem can obviously be solved by ad hoc means.

\section{Experience with Transactions as Objects}

As expected, object-based implementation of transactions permits significant code reuse. Our implementation successfully reused MELD's name service and interprocess message passing facility. About 160 lines of message passing interface code were adapted to reuse "as is" approximately 800 lines of runtime support code and the 2400 lines of the name server in passing messages between transaction "objects". Implementing transactions as first-class MELD objects, rather than as simulated "objects" and "methods" (the C transaction structures and associated operations that we actually used), would most likely permit additional reuse of the object management and method invocation code.

We have also found that transaction objects permit design as well as code reuse. We have generalized the framework of transaction objects, and their local concurrency control and global distributed commit protocol operations, to support the standard two-phase locking protocol.

In our preliminary design, transactions continue to be represented as simulated "objects" by C structures with essentially the same local and global operations as for the optimistic concurrency control algorithm. The data structures for two-phase locking closely resemble those already implemented for the optimistic algorithm, and the changes are mainly confined to the concurrency control mechanism (local operations) rather than the commit protocol (global operations). To avoid having to add lock data to individual objects, replacing the multiple versions of instance variables for the optimistic mechanism, the preliminary design uses a separate lock manager "object' for each process. The relevant data structures are shown in Figure 7-1. 
Because of this decision, no fields need be added to transaction objects to implement two-phase locking, per se; a locking transaction structure can be more or less the same as an optimistic transaction structure, with the optimistic concurrency control fields (g_sn through write_set in Figure 4-1) deleted. In our design, we have added one field, bk_versions; this is not necessary for concurrency control, but allows recovery in case a deadlock is detected and the transaction must be aborted. The bk_versions field contains a list of pairs of changed variables and their old values; in case of an abort, the variables would be restored to their backup values.

The lock manager in the preliminary design maintains two tables, one organized by object and searched to determine whether a lock exists on a particular object (or instance variable, depending on the granularity selected), and one organized by transaction and used at commit or abort time to free all locks belonging to the transaction. The lock table is represented as a structure holding these two lists. In a realistic implementation, these tables should not actually be linked lists, but should be implemented more efficiently. Here, however, we treat them as (separate) lists, since we are concerned only with the design and not the details of an implementation. The transaction and lock table data structures for two-phase locking are shown in Figure 7-1.

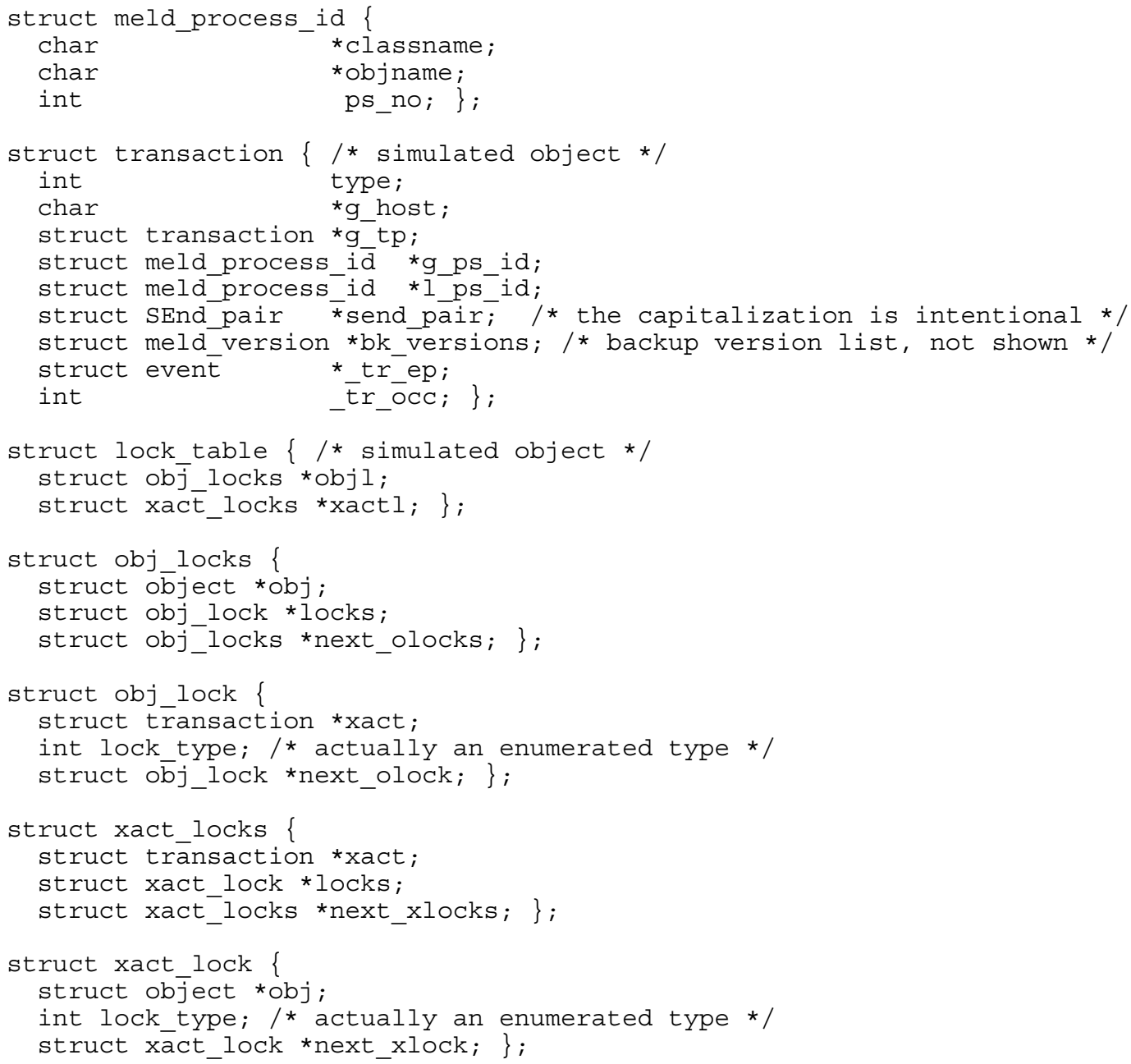

Figure 7-1: Locking Transaction System Data Structures 
There are three local concurrency control operations for two-phase locking transactions: Obtain a read lock, obtain a write lock, and release all locks belonging to a transaction. The lock manager provides these operations, but they are routed through the transaction "object' to provide a uniform transaction interface and to allow the transaction to keep track of changes in its bk_versions list. Figure 7-2 shows the local and global operations for two-phase locking, using the same names as the optimistic algorithm and similar interfaces wherever possible. One local operation, to clean up the locks left at a cohort process by a transaction, has been added; two, for version maintenance of instance variables for the optimistic concurrency control algorithm, have been removed. (In a multi-version locking scheme, these two operations would return; however, since our initial implementation will not use multiple versions, these local operations are not needed.)

\section{Local Two-Phase Locking Operations}

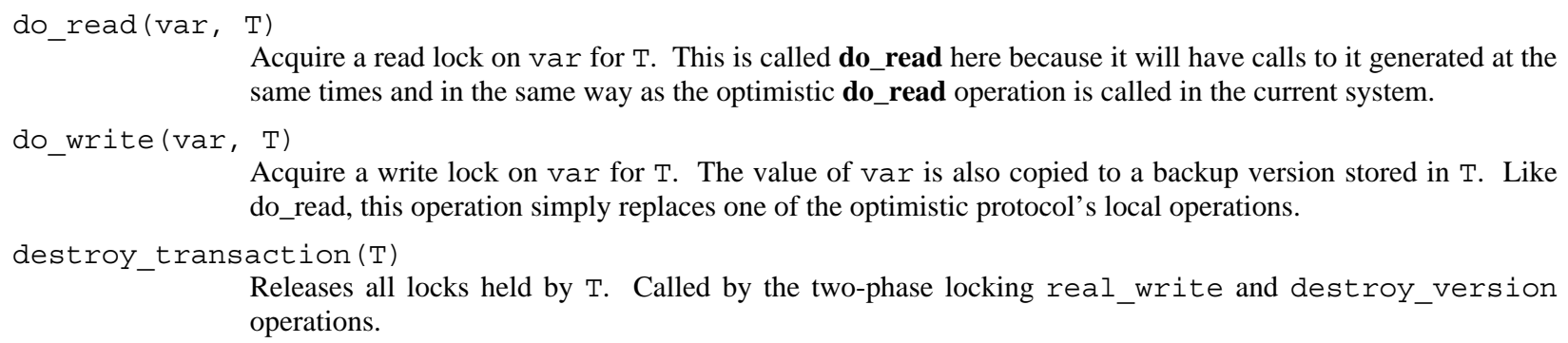

Acquire a read lock on var for T. This is called do_read here because it will have calls to it generated at the same times and in the same way as the optimistic do_read operation is called in the current system.

Releases all locks held by T. Called by the two-phase locking real_write and destroy_version operations.

\section{Global Distributed Commit Protocol Operations}

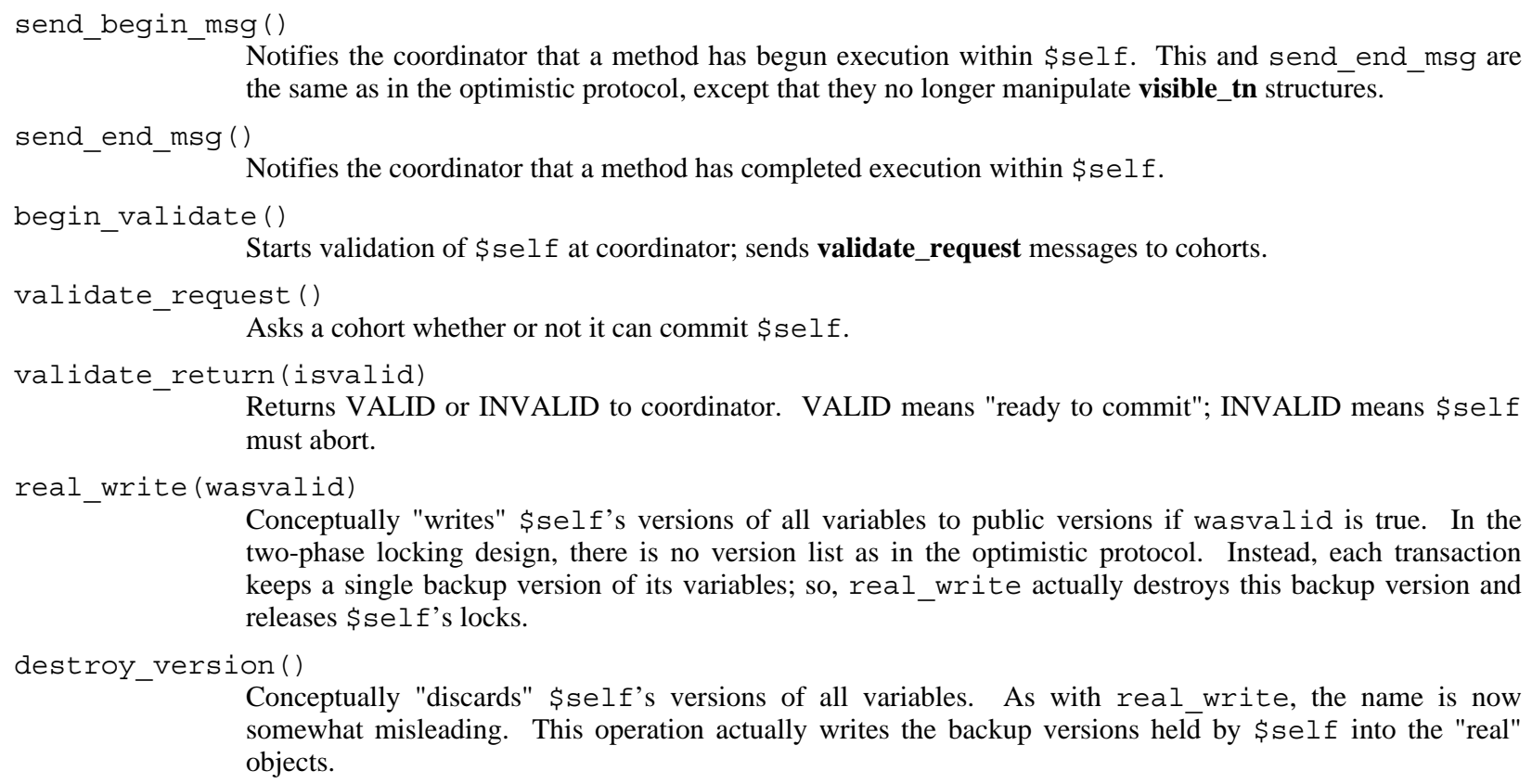
somewhat misleading. This operation actually writes the backup versions held by \$self into the "real" objects.

Figure 7-2: Operations for Two-Phase Locking

The interface to the global operations of termination detection and the commit protocol remain the same except for the dropping of some now unnecessary arguments. The message types sent also remain the same, and in the same order, as in Figures 5-2 and 5-3, although their names are 
now less descriptive. Their implementations change as described in Figure 7-2. This design allows two-phase locking to be substituted for MELD's optimistic protocol, without any other changes to MELD's implementation.

\section{Related Work}

A few innovative engineering- or design-oriented systems represent development tasks as objects (e.g., [3]). The purpose of these objects is to provide a persistent developer-visible "audit trail" of changes to the system under development and allow reasoning about these changes. Most of these systems are not concerned with reuse of concurrency control design or implementation, and in many cases are not concerned with concurrency control at all.

One notable exception is Cosmos [13], an operating system facility intended to support multiuser software development environments. Cosmos provides an explicit notion of transaction objects, which represent in-progress development tasks. A transaction object includes information about the task such as the new versions of changed development objects created during the task, and when committed turns into a persistent configuration object that represents a consistent state of the system under development. Concurrency control and recovery in Cosmos are implemented in terms of these transaction objects and configuration objects, in that a transaction object collects the new versions private to the development task until it is completed, and it is always possible to recover back to a previous configuration object. But there is no notion analogous to MELD's message passing among the objects in order to implement these mechanisms.

Avalon does use message passing within an object to implement concurrency control on that object, although there is no notion analogous to Cosmos's and MELD's objects representing transactions on other objects. Avalon provides a set of system-defined superclasses whose methods represent primitive operations for constructing synchronization and recovery mechanisms. This gives the programmer an object-oriented facility for defining new classes of objects with desirable properties, such as atomicity and persistence.

\section{Conclusions}

Our implementation of distributed concurrency control for MELD has provided some insights into the problems of transactions on objects, specifically their interactions with and impact on the message passing facility, concurrent non-transactional code, and object management operations. More significantly, treating transactions as primitive "objects" has allowed us to reuse some internal MELD facilities in the implementation of the transaction subsystem, and promises to allow reuse of design in implementations of alternative concurrency control mechanisms. There do not seem to be any particular aspects of our implementation of transaction "objects" that are peculiar to MELD, so this technique should also be useful for other distributed object systems.

Using transaction objects in our implementation enabled us to reuse MELD's existing communication mechanisms, which account for a significant fraction of MELD's runtime code. In our design for implementing an alternative concurrency control mechanism, two-phase locking (discussed in Section 7), we have reused and generalized the design for the transaction 
object mechanism. Transaction objects show great promise as an implementation strategy for reusable, extensible concurrency control in object systems.

\section{Acknowledgments}

Wenwey Hseush worked with the authors on the design and implementation of the previous version of concurrent MELD, without transaction blocks. David Staub developed MELD's name service. Quoc-Bao Nguyen ported MELD from Sun-3 under SunOS 4.0 to Sun-4, IBM RT under AIX 2.2.1 and Mach 2.5, and DecStation 3100 under Ultrix 3.1. David Garlan worked with Gail Kaiser on the initial development of a sequential version of the MELD language. We would like to thank Travis Winfrey for his comments on an earlier draft of this paper.

\section{References}

[1] D. Agrawal, A.J. Bernstein, P. Gupta and S. Sengupta.

Distributed Optimistic Concurrency Control with Reduced Rollback.

Journal of Distributed Computing 2(1):45-59, April, 1987.

[2] Philip A. Bernstein, Vassos Hadzilacos and Nathan Goodman.

Concurrency Control and Recovery in Database Systems.

Addison-Wesley, Reading MA, 1987.

[3] Don Cohen and K. Narayanaswamy.

A Logical Framework for Cooperative Software Development.

In 6th International Software Process Workshop. October, 1990.

Preprints.

[4] David Detlefs, Maurice Herlihy and Jeannette Wing.

Inheritance of Synchronization and Recovery Properties in Avalon/C++.

Computer 21(12):57-69, December, 1988.

[5] Wenwey Hseush and Gail E. Kaiser.

Data Path Debugging: Data-Oriented Debugging for a Concurrent Programming Language.

In ACM SIGPLAN/SIGOPS Workshop on

Parallel and Distributed Debugging, pages 236-246. Madison WI, May, 1988.

[6] Gail E. Kaiser and David Garlan.

Melding Software Systems from Reusable Building Blocks.

IEEE Software 4(4):17-24, July, 1987.
[7] Gail E. Kaiser and David Garlan.

MELDing Data Flow and Object-Oriented Programming.

In Object-Oriented Programming Systems, Languages and Applications Conference, pages 254-267. Orlando FL, October, 1987.

[8] Gail E. Kaiser, Steven S. Popovich, Wenwey Hseush and Shyhtsun Felix Wu.

MELDing Multiple Granularities of Parallelism.

In 3rd European Conference on ObjectOriented Programming, pages 147-166. Cambridge University Press, Nottingham, UK, July, 1989.

[9] Gail E. Kaiser, Wenwey Hseush, Steven S. Popovich and Shyhtsun F. Wu. Multiple Concurrency Control Policies in an Object-Oriented Programming System. In Second IEEE Symposium on Parallel and Distributed Processing. Dallas TX, December, 1990.

In press.

[10] H. T. Kung and John Robinson.

On Optimistic Methods for Concurrency Control.

ACM Transactions on Database Systems 6(2):213-226, June, 1981. 
[11] Subrata Mazumdar and Aurel A. Lazar.

Knowledge-Based Monitoring of Integrated Networks.

In Branislav Meandzija and Jil Westcott (editors), IFIP TC 6/WG 6.6 Symposium on Integrated Network Management, pages 235-243. North-Holland, Boston MA, May, 1989.

[12] David P. Reed.

Naming and Synchronization in a Decentralized Computer System.

$\mathrm{PhD}$ thesis, MIT, September, 1978.

MIT LCS TR-205.

[13] J. Walpole, G.S. Blair, J. Malik and J.R. Nicol.

Maintaining Consistency in Distributed Software Engineering Environments.

In 8th International Conference on

Distributed Computing Systems, pages

418-425. San Jose, June, 1988. 